\title{
Unexpected diversity of cnidarian integrins: expression during coral
} gastrulation

\author{
Brent A Knack ${ }^{\dagger 1}$, Akira Iguchi ${ }^{\dagger 1}$, Chuya Shinzato ${ }^{1}$, David C Hayward ${ }^{2}$, \\ Eldon E Ball*2 and David J Miller*1
}

Address: ${ }^{1}$ ARC Centre of Excellence for Coral Reef Studies, James Cook University, Townsville, Queensland, 4811, Australia and ${ }^{2}$ Centre for the Molecular Genetics of Development and Research School of Biological Sciences, Australian National University, P. O. Box 475 Canberra, ACT, 2601, Australia

Email: Brent A Knack - brent.knack@jcu.edu.au; Akira Iguchi - akira.iguchi@jcu.edu.au; Chuya Shinzato - chuya.shinzato@jcu.edu.au; David C Hayward - david.hayward@anu.edu.au; Eldon E Ball* - eldon.ball@anu.edu.au; David J Miller* - david.miller@jcu.edu.au

* Corresponding authors †Equal contributors

\section{Published: 9 May 2008}

BMC Evolutionary Biology 2008, 8:136 doi:10.1/86/147|-2/48-8-136
Received: 26 October 2007

Accepted: 9 May 2008

This article is available from: http://www.biomedcentral.com/I47I-2/48/8/I36

(c) 2008 Knack et al; licensee BioMed Central Ltd.

This is an Open Access article distributed under the terms of the Creative Commons Attribution License (http://creativecommons.org/licenses/by/2.0), which permits unrestricted use, distribution, and reproduction in any medium, provided the original work is properly cited.

\begin{abstract}
Background: Adhesion mediated through the integrin family of cell surface receptors is central to early development throughout the Metazoa, playing key roles in cell-extra cellular matrix adhesion and modulation of cadherin activity during the convergence and extension movements of gastrulation. It has been suggested that Caenorhabditis elegans, which has a single $\beta$ and two $\alpha$ integrins, might reflect the ancestral integrin complement. Investigation of the integrin repertoire of anthozoan cnidarians such as the coral Acropora millepora is required to test this hypothesis and may provide insights into the original roles of these molecules.

Results: Two novel integrins were identified in Acropora. Amltgal shows features characteristic of $\alpha$ integrins lacking an I-domain, but phylogenetic analysis gives no clear indication of its likely binding specificity. Amltg $\beta 2$ lacks consensus cysteine residues at positions 8 and 9 , but is otherwise a typical $\beta$ integrin. In situ hybridization revealed that Amltg $\alpha$ I, $A m \operatorname{ltg} \beta I$, and $A m \operatorname{ltg} \beta 2$ are expressed in the presumptive endoderm during gastrulation. A second anthozoan, the sea anemone Nematostella vectensis, has at least four $\beta$ integrins, two resembling Amltg $\beta$ I and two like Amltg $\beta 2$, and at least three $\alpha$ integrins, based on its genomic sequence.

Conclusion: In two respects, the cnidarian data do not fit expectations. First, the cnidarian integrin repertoire is more complex than predicted: at least two $\beta$ s in Acropora, and at least three $\alpha$ s and four $\beta$ s in Nematostella. Second, whereas the bilaterian as resolve into well-supported groups corresponding to those specific for RGD-containing or laminin-type ligands, the known cnidarian as are distinct from these. During early development in Acropora, the expression patterns of the three known integrins parallel those of amphibian and echinoderm integrins.
\end{abstract}

\section{Background}

Integrins are a large family of cell surface transmembrane receptors known only from metazoans, which function in intracellular signalling as well as cell-cell and cell-extracel- lular matrix (ECM) adhesion [1]. As the main mediators of cell-ECM interactions they are key players in early development [2] functioning in gastrulation by rapid modulation of their own adhesion between low and high 
affinity states and by modulating the activities of adhesion molecules (e.g. cadherins) in cell layers undergoing convergence and extension [3].

Integrins function as $\alpha \beta$ heterodimers with several subunits of each type being present in most animals. Analyses of the whole genome sequence of Caenorhabditis elegans $[4,5]$ indicate that it has a single $\beta$ subunit of the $\beta 1$ type that is capable of associating with two $\alpha$ subunits, which confer specificity for either laminin- or RGD-containing ligands, and it has been suggested that this may reflect the ancestral state. Drosophila melanogaster has five $\alpha$ and two $\beta$ subunits [5] and mammals, eighteen $\alpha$ and eight $\beta$ integrin subunits [6]. In each case, however, integrin subunits above and beyond likely orthologs of the two $\alpha$ s and one $\beta$ of Caenorhabditis are clearly lineage-specific. "Lower" animals are of particular significance in terms of understanding the ancestral state, but have not been extensively studied. Both $\alpha$ and $\beta$ integrin subunits have been identified in sponges [7-9], and cnidarians $[7,10,11]$, but the extent of integrin diversity and the range of functions of these molecules in "lower" animals are unknown.

Anthozoan cnidarians such as the coral Acropora millepora and the sea anemone Nematostella vectensis appear to have retained much of the genetic complexity of the metazoan common ancestor $[12,13]$; hence these animals are likely to be highly informative with respect to the ancestral integrin complement and may provide insights into the original roles of these molecules. Known cnidarian integrins include a $\beta$ integrin from Acropora millepora [7] and single $\alpha$ and $\beta$ subunits (IntA and IntB) from the hydrozoan jellyfish Podocoryne carnea [11]. Here we report the characterisation of novel $\beta$ and $\alpha$ integrins from Acropora. The known Acropora integrins are expressed during gastrulation in patterns like those seen at the corresponding stages of echinoderm and amphibian development. However, we know from morphological observation $[14,15]$ that Acropora gastrulation is not a simple epithelial to mesenchymal transition in which the expressing cells lose their adhesivity and invaginate. Instead, in Acropora it is clear that changing cell shape also plays a major role[14]. Two further implications of this work are that the cnidarian integrin complement is significantly more complex than was predicted, and that functional diversification of $\alpha$ integrins may have occurred independently in Cnidaria and Bilateria.

\section{Results}

Identification of novel integrins

Three unigenes encoding integrin subunits were identified during an ongoing EST analysis of Acropora millepora $[13,16]$. One of these corresponds to the previously known Acropora integrin $\beta \mathrm{Cn} 1$ [7]; to simplify compara- tive analyses, this integrin is henceforth referred to as AmItg $\beta 1$. Complete sequences were determined for cDNA clones corresponding to the other two integrin unigenes; comparative analyses indicated that an EST clone corresponding to a second $\beta$ subunit ( $A m I \operatorname{tg} \beta 2$ ) encodes a full length protein of 771 amino acids, whilst an $\alpha$ integrin EST clone lacked the $5^{\prime}$ end of the open reading frame. To complete the 1021 amino acid integrin $\alpha$ coding sequence, overlapping clones were isolated from a cDNA library, enabling the determination of the complete open reading frame for a molecule designated AmItg $\alpha 1$. These sequences have been submitted to GenBank under the following accession numbers: EU239371 (AmItg $\alpha 1$ ) and EU239372 (AmItgß2).

\section{Amltg $\beta 2$ is a possible coral ortholog of a known jellyfish integrin $\beta$}

Database comparisons identified $\operatorname{AmItg} \beta 2$ as a possible ortholog of integrin $\beta$ (IntB; Q9GSF3) from Podocoryne [11]. Previously Reber-Muller et al. [11] suggested that Podocoryne IntB was orthologous with AmItg $\beta 1$; however, the former not only has higher overall sequence identity with $\operatorname{AmItg} \beta 2$ ( $44 \%$ amino acid identity compared to $<40 \%$ ), but also shows the same atypical pattern of cysteine residues (Fig. 1,2) Whereas the $\beta$ integrin extracellular domain characteristically contains 56 cysteine residues arranged in a specific pattern [7], in both Podocoryne IntB [11] and AmItg $\beta 2$, cysteine residues at positions 8 and 9 in the canonical structure are absent as occurs in vertebrate $\beta 4$-type integrins. In terms of most other structural features, however, both AmItg $\beta 2$ and Podocoryne IntB are typical integrin $\beta s$ - the MIDAS domain, cysteine-rich stalk and transmembrane region are all clearly present. In both cases, the DxSxS motif of the MIDAS cation-binding domain [17] is completely conserved, whereas the DDL motif of the ADMIDAS is changed to EDL in AmItg $\beta 2$ (Fig. 1). The cytoplasmic domains contain the conserved membrane proximal sequence KLLxxxxD and two NPxY/F motifs (NPIF and NPTY in AmItgB2, NPIY and NPMY in IntB). Whereas the degree of similarity between these Acropora and Podocoryne sequences implies that these might be orthologs, one complicating factor is that the Nematostella genome appears to encode two integrins of the AmItg $\beta 2$ type (see below). Hence orthology relationships will only be clear when more complete datasets are available for Acropora and Podocoryne.

\section{Amltg $\alpha$ I is a cnidarian integrin resembling the vertebrate $\alpha$ 4/9-type}

Comparative analyses indicate that AmItg $\alpha 1$ shares some characteristics with those integrin $\alpha$ subunits that lack an $\alpha-A$ (I) domain. Database comparisons revealed that AmItg $\alpha 1$ is most similar to mouse integrin $\alpha 9$ (MmItg $\alpha$; Q91YD5; 28\% identity and 48\% similarity). Whereas the Acropora and Podocoryne $\beta$ integrins that are possible 


\begin{abstract}
AmItg $\beta 2$ $\operatorname{AmItg} \beta 1$ PcIntB

HsItg $\beta 1$ SpItg $\beta$ G SpItg $\beta$ L OtItg $\beta 1$

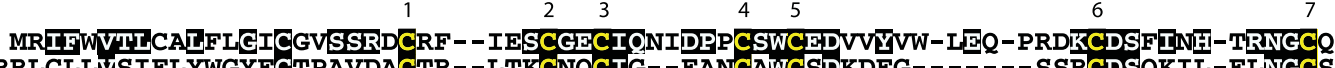
MKRRLCLLVSIFLYWGYFCTPAVDACTR--LTKCNQCIG--EANCAWCSDKDFG------SSRCDSQKIL-ELNGCS MHFFQIISILILHUGYIKFTAAVHECNT--KLTCSDCIS-SSPKCSWCEDIYYNK-TEKYFHKKCDLVETIT-QRQLCS MNLOPIFWIGLISSVCCVFAOTDENRCLKANAKSCGECIO-AGPNCGWCTNSTFLQ-EGMPTSARCDDLEAL-KKKCCP MAWKCLLVLTIAFSAGQIYRTYAQTSSSACSD--AKTCGECIS-LDSSCGWCTLLN-- YTDDTGNPQCDLASSL-SQRGCS

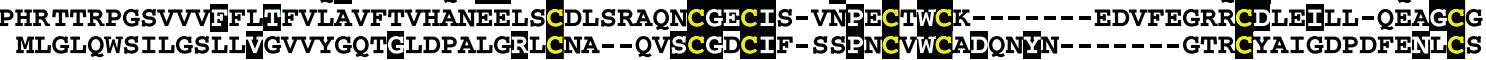
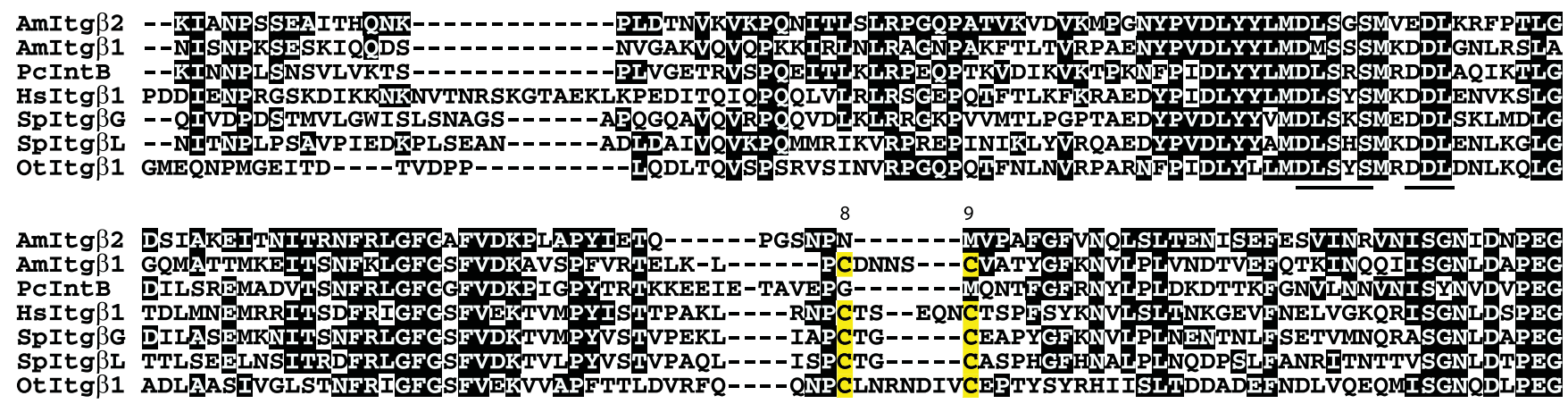

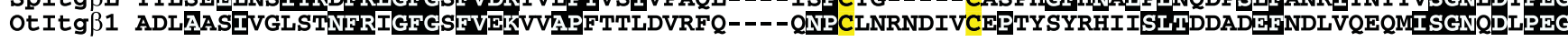

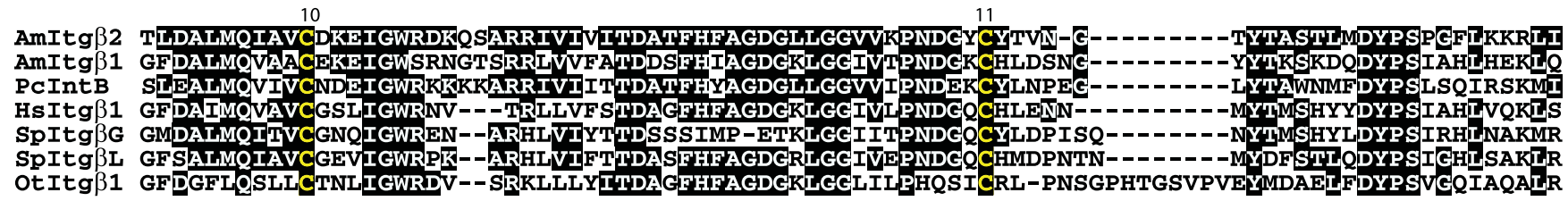
OtItg $\beta 1$ GFDGFLOSLLCTNLIGWRDV--SRKLLLYITDAGFHFAGDGKLGCLILPHQSICRL-PNSGPHTGSVPVEYMDAELFDYPSVEQIAQALR

AmItgß2 DEQVSPIFAINQSK-VDVYRQYOFIVTFFGSESGAVSESTRIDSSNLLQLIROAYEKIAKTQTIRD-PAPEGVKVNYTAYCP-WIGKKVGS AmItg $\beta 1$ ESNVLPIFAVTKQ----FASLYKSVSTMWS-DLGAVTGTLATDSDNVVELIKNKYEEIVSTVSLVY -KEPERVSVTVKANCGPNSVNTQT PCIntB ENQMIPIFATTGN----TALYQKVAEFFGEASGAVADALNSNSSNVVPIIKDAYQKIAKMVSIKA-DASDEIDVKFQAMCG-KEELKDS HsItg $\beta 1$ ENNIQTIFAVTE----EFQPVYKFLKNLIP---KSAVGTLSANSSNVIQLIIDAYNSLSSEVILENGKLSEGVTISYKSYCKNGVNGTEE SpItg $\beta$ G ENSVIPIFAV----IOKEFEIYNNITQYIE--GATAG-IIAQDSNNIVQLVKDNYSKITSRVEVVD-DAPENVTIDYGPHCPGGQVTPGS SpItg $\beta L$ ENNVIPIFAV----TRDQTPLYMSLEKDIE--GATVG-TLDIGSGNVVQLIRSNCDRITSQVRLTS-TAPDDVTLSYRANCKD-QTYQDT OtItg 31 EQDIIPIFAAQR---DAREFYDVLAAEIG--EGASTGTLASDSSNVVELVRQQYNTISQRVIFDQEPVPGVSVVINPTSCPGGVIEDE-

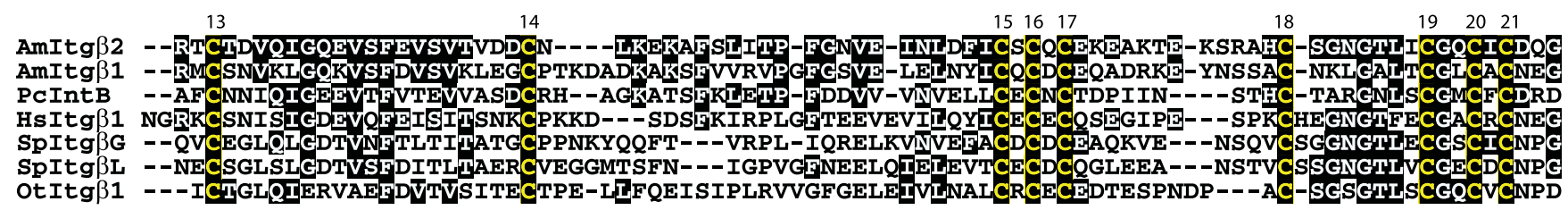

Figure I

$\beta$ integrin alignments (amino terminal end of the molecules). Amino acid sequence of Amltg $\beta 2$ aligned with representative $\beta$ integrin sequences. Atypical absence of cysteines (yellow, numbered) from positions 8 and 9 suggests orthology between Amltg $\beta 2$ and Podocoryne IntB (PclntB). Structural features including the MIDAS motif (DLSXS, underlined), transmembrane region (long wavy line), membrane proximal motif (short wavy line), and two NPxY/F motifs (double underline) are conserved. The ADMIDAS motif (DDL, underlined) is changed to EDL in Amltg $\beta 2$. An arrow indicates the position where a deletion was made in the sponge sequence (Otltg $\beta I$ ) to facilitate alignment. Abbreviations and database accession numbers for sequences used in the alignment are: Acropora Amltgß2 (Amltgß2; EU239372); Podocoryne IntB (PclntB; AAG25994); Acropora Amltg $\beta$ I (Amltg $\beta$ I; $\underline{A A B 66910}$ ); Human $\beta$ I (Hsltg $\beta$ I; P05556); Strongylocentrotus $\beta$ G (Urchin Spltg $\beta$ G; $\underline{A A B 39739}$ ); Strongylo-

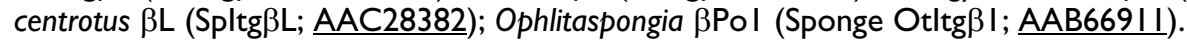

orthologs (AmItg $\beta 2$ and PcIntB) are $44 \%$ identical, the $\alpha$ subunits AmItg $\alpha 1$ and Podocoryne IntA have a much lower amino acid identity (27\%), and there are several differences between these that are likely to have functional and/ or structural significance. Both proteins are typical in terms of the presence of FG-GAP repeats, transmembrane regions, membrane proximal KxGFFKR motifs and extracellular cleavage sites fitting the RxK/RR consensus (Fig. 3,
4). However, whereas AmItg $\alpha 1$ is typical in having three cation binding motifs (DxD/NxD/NxxxD; [18]) within FG-GAP repeats $\mathrm{V}, \mathrm{VI}$, and VII, the three cation binding sites in Podocoryne IntA are in FG-GAP repeats VI, VII and immediately C-terminal of repeat VII. Both proteins are atypical in terms of the positions of cysteine residues relative to the consensus; AmItg $\alpha 1$ is missing Cys residues at positions 9, 10 and 17, whereas IntB is missing Cys resi- 

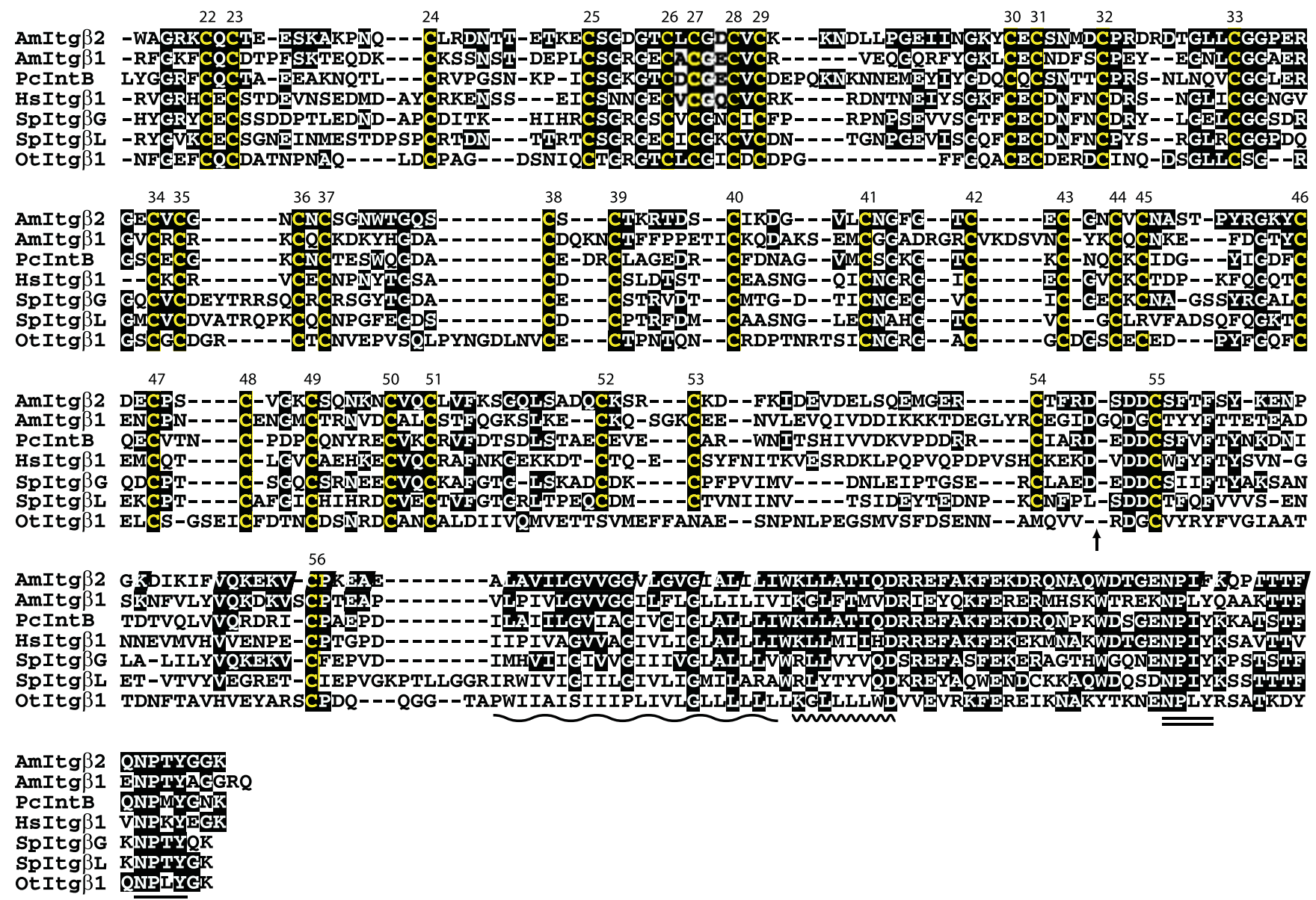

Figure 2

$\beta$ integrin alignments (carboxy terminal end of the molecules). See legend for Fig. I.

dues at positions 13 and 14, but both proteins have a novel Cys pair between consensus positions 10 and 11. A corresponding extra pair of Cys residues is also present at the same position in both the Nematostella $\alpha$ integrin predicted from the genome sequence (NvItg $\alpha 1$; see below) and the atypical Drosophila integrin PS3.

\section{Phylogenetic analyses of the novel integrin sequences}

To better understand relationships between the Acropora sequences and the major integrin types of higher animals, maximum likelihood (ML) phylogenetic analyses were undertaken using MolPhy version 2.3 [19]. Integrin phylogenetics is complicated by high levels of primary sequence divergence and homoplasy, leading to difficulties in unambiguous alignment of sequences. The analyses presented here are therefore based on extensively edited alignments. Sequences were aligned using ClustalW via the EBI website, manually edited using JalView, and used for phylogenetic analyses. In the case of the integrin $\alpha$ alignment, the output from ClustalW consisted of 2383 positions and was manually edited to 1091 posi- tions (20 sequences). The corresponding figures for the integrin $\beta$ alignments were 2159 positions prior to editing and 991 after editing (24 sequences).

The ML phylogenetic analyses of integrin $\alpha$ sequences (Fig. 5A) are broadly consistent with previous studies; the resolution of bilaterian sequences into two major clades corresponding to the major ligand classes RGD (PS2) and laminin (PS1) is strongly supported. The fact that these two clades each contain protostome (fly, worm) and deuterostome (human, sea urchin) sequences indicates that the functional divergence of $\alpha$ integrins had already occurred in Urbilateria - the common ancestor of bilateral (higher) animals. However, the ML phylogenetic analyses give no clear indication of the likely ligand specificity of the known cnidarian $\alpha$ integrins. The cnidarian (Podocoryne, Acropora and Nematostella) $\alpha$ integrins group together with high bootstrap support (albeit on long braches which reflects their divergence), the sister group of this cnidarian integrin $\alpha$ clade being the $\alpha 4 / 9$ type integrins (Fig. 5A). 


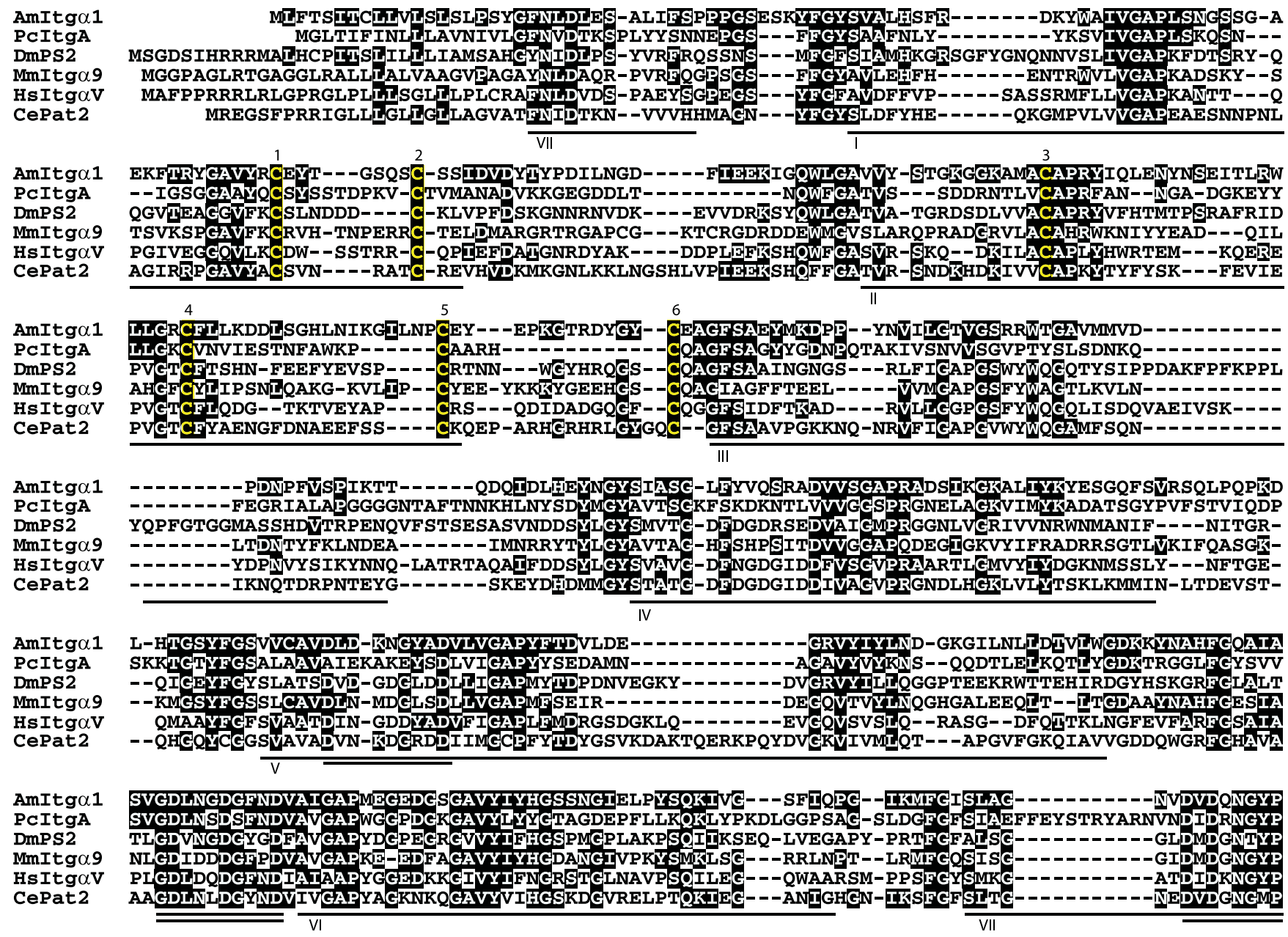

\section{Figure 3}

$\alpha$ integrin alignments (amino terminal end of the molecules). The major structural features of alpha integrins lacking an alpha-A domain are conserved in Amltg $\alpha$ I including seven FG-GAP repeats (underlined, roman numerals), three DxD/ $\mathrm{NxD} / \mathrm{N} x x \times \mathrm{D}$ cation binding sites (double underline), the transmembrane region (long wavy line) and the cytosolic membrane proximal domain (short wavy line). The position of a putative fourth cation binding site in the Podocoryne sequence is indicated in red. Arrows mark the positions where regions that could not be unambiguously aligned were removed from the Drosophila (DmPS2; 219 residues), Caenorhabditis (CePat2; I 32 residues) and human (HsItg $\alpha$ V; 6 residues) sequences. Abbreviations and

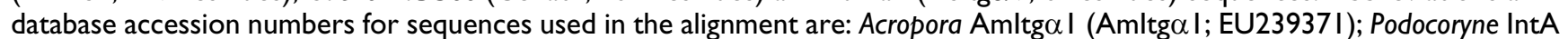
(PclntA; AAG25993); Drosophila $\alpha$ PS2 (DmPS2; PI2080); Mouse $\alpha 9$ (Mmltg $\alpha$ 9; NP 598482); Human $\alpha \mathrm{V}$ (Hsltg $\alpha \mathrm{V} ;$ P06756); Caenorhabditis aPat2 (CePat2; P34446).

ML analyses of the integrin $\beta$ sequences (Fig. 5B) confirmed the possible orthology of Podocoryne IntB with the novel Acropora $\beta$ sequence (AmItg $\beta 2$ ) reported here. Preliminary surveys of the genome sequence of Nematostella suggest the presence of at least four $\beta$ integrins, and gene models (the gene as predicted in the genome assembly, including the open reading frame, introns and untranslated regions) of these were sufficiently complete for them to be included in phylogenetic analyses (Fig. 5B) which group the Nematostella $\beta$ integrins with the Acropora subu- nits - two with AmItg $\beta 1$ and two with AmItg 32 . It remains to be seen whether each member of these pairs of Nematostella genes has an Acropora ortholog. As in the integrin $\alpha$ phylogeny, the sponge sequences were relatively distant to those from the Cnidaria. Again, the analyses were broadly consistent with previous studies [20$22]$, resolving the vertebrate sequences into three clades known as $\beta 1$ (integrin $\beta 1 / 2 / 7$ ), $\beta 3$ (integrin $\beta 3 / 5 / 6 / 8$ ) and $\beta 4$ in the Hughes [20] phylogeny. Unlike the $\alpha$ integrins, there is no evidence for divergence of $\beta$ subunits 
AmItga1 PcItgA DmPS2 MmItg $\alpha 9$ HsItg $\alpha$ V CePat2

AmItg $\alpha 1$ PcItgA DmPS2 MmItg $\alpha 9$ HsItg $\alpha$ V CePat2

AmItg $\alpha 1$ PcItgA DmPS2 MmItg $\alpha 9$ HsItg $\alpha$ V CePat2

AmItg $\alpha 1$ DmPS2 HsItg $\alpha$ V CePat2

AmItg $\alpha 1$ PcItgA DmPS2

MmItga 9 HsItg $\alpha$ V CePat2

AmItg $\alpha 1$ PcItgA DmPS2

MimItg $\alpha 9$ HsItg $\alpha$ V CePat2

AmItg $\alpha 1$ PcItgA DmPS2

MimItg $\alpha 9$ HsItg $\alpha$ V CePat2

AmItga1 PcItgA
DmPS2

MimItg $\alpha 9$ HsItg $\alpha$ V CePat2

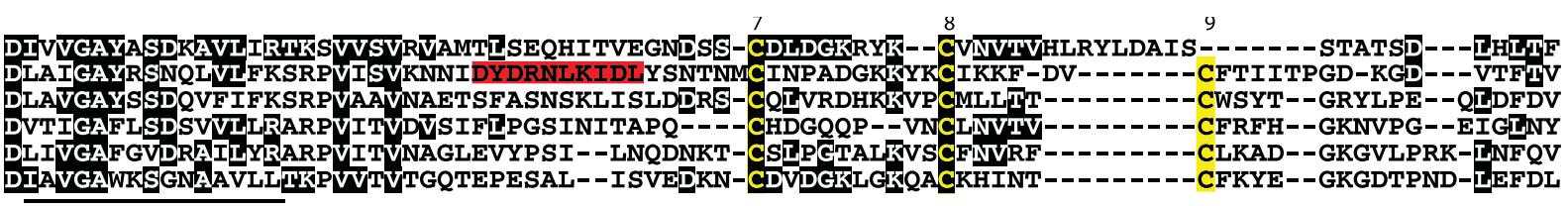

10

TFDLD- - - - - --KDIDVQLRRMFFYDEVTKSRTVSLRQNTTLAKPNTTYEVLRKTAYIKSKDELGDLTSLIPFEVTLSQPQP- - - DID----------KETTGGKRGFVVEGTQQKSVFSGVVTEI----AQVAKCQPYIIYLRNN--TKDVFREMIIKISWTLKQP----SWL---------LDAKKLLNPRMFFLRDEGKNIRNQTIRLN----YYGQKYCLNETVYLLD--KVQDKLTPIEVEARYNLRSSRPLDP NLTAD--------VAQKEKGQLPRVYFVLFGETAGQVSERLQLS---HMDEVCHHYVAHVKRR--VQDVISPIVFEAAYSLDEHVIMGE-

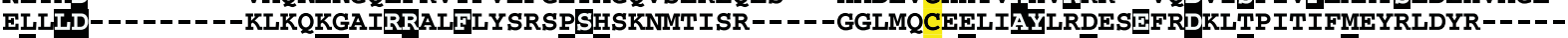
RFNLDDHSPEPRAYFLQKDVKSDRSIKVAQGSTTRDHPSSIEQRVRLEKGRQKCFRHRFFASST--MKDKLSPIHWSV̄NYTYVES----K PcItgA

MmItga9

11 -CRTIRI - - 12

12

ISARPFNPKSD---VIRLEVVKDFTIFLTVENKAFDSAYTSRI NSDLSIVMIKQLAGDFKT---IQVGITKTLNIVAKITMKMMIEN-AFINTL

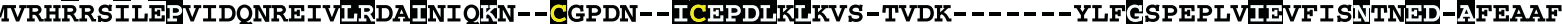
-EDRELPDLTPVIRWKKGQRISQKNQTVFERNCQSED---CAADIQLIRGKLLLSSVDEKTPHLALCAVKNISLNISISNLGDD-AYDANV -TAADTTGLQPILNQFTPANISRQAHILID--CGEDN--VCKPKLEVSVDSDQKK--- - - IYIEDDNPLTLIVKAQNQGEG- AYYEAEL TGKLRGDKLEPAIDTTVPLSFQNKINIA ANN--CGKDD--LCVPDLKVTA3VADREK-----FULGTQDNTMLINVTVQNGGED-SYETKL

13

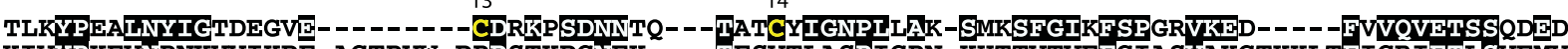
KIVYPKEVNPNKVVIKDF-AGTPVW-DPDSTKDGNEK----TFSVTLASPIGPN-KHTTVTVEF GIASVAKGTKKLTEIGRIETISVEMV YMVTPPDIOFRKLOOLGEKKDTPIT--CSPPTPENNH----TLKCDIGNPLESG-KIAHFKISLVPEEKYGSSSSYDFYWEANSTNLEKP SFNVSRELFFINMWOKEV---MGIS--CELLESD-----FLKCSVGF PFMRSKSKYEFSVIFDTSHLSGEEEILSEIVTAOSGNLERS IVSIPLQADFIEVVRNNE-ALARLS--CAFITTENQTR----QVVCDLGNPMKAG-TQLLAGLRFSVHQQS TMDTSVKFDIQIQSSNLFDK YFDVPQGFEYGGIESVGGDGSKSAPA-CSPTSDEPDSDGKWTFACDLGNPIPAN-KVVSSVVRVTASSDKPPLAPISINAHVNSSNDEFA

ANIFDNKKTISVAVKYEADVEITGTTKQDQVVYQGPVRS- - --KEFVKKDLDSIGPEIVQTLSVRNINPSDIRSSELVVSFPKAYS-SS PAD--NVYTLEIPVSLLANLTVTENVEPEQLKWNKDASP---------KDVEAIEIIHTFYFQNLGPSTVDTSDVVIQFPER----GSEYDNKIROSVGIWVDTDLDIKGTSLPDYOLYKADDYK-ELENATKE----DDIGPOVVHIYEIRNNRPSITEEAEVFIHLPY----ET EALHDNTLTLTVPLVHFVDTSITEIVSPTSFVYGESVDDASN----FIQLDDOECHFOPVNITLOVYMMIGPSTLPGSSVSISFPS---RLS

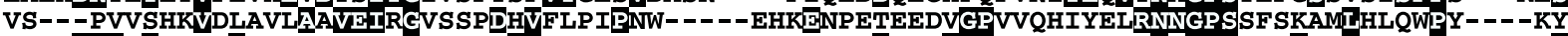
HTVADNKVFTTIPVDFKNQLSLNERSNP EQVDF SMTNK----TRVDAFDDNEIGPVVSHLYQISMRGPSEVDSATLDIFWBS--- FI

KPDSYLLYLLLVELDGAS-----GIC----DASVNPLKIKPRNEWATESTPSRRRRD-------------TGNLVLSC--RQAAASQTFKKC FDGSVMFNIFKADLKGTDKSRSVGTCT---Y YKLNTLGLNVTTEEVGGVNSTAKKLRARRAAD--------TIPOLGC-GGSIKCREIKC IVGDPIMYLLNQPETGG----KIOCDEKARGEGFVRGVLVS SSTDAGDKLSPKOVEQRRQEDTLEALGLQIDLNSPCOSAR--CKSIRC PGGAEMFOVQDMVVSQ-----EKGNC--SLQRNPTPCIIPQEO--ENIFHTIFAFFSKSGRK-----------VLDCEKPGSFCLTLHC N-NNTLLYILHYDIDGP------MNC--TSDMEINPLRIKISS-LQTTEKNDTVAGQ-GERDHLITKRD--DIHTLGC--GVAQCLKIVC TEGGHLLYIITEPVVNPP---NKGRCRVKQLQNVNPLNLRITNEHVPTEPPVAKTPNEYSREEDDESYEPLSRASVDC--NSLRCTHIEC $\uparrow$

QLGQLKAGDKAN-TKMTFR--FW̄EN-TLLKELDS---PKAVELVTSANVKVSDDITQSNYENDDTEIKIKAPRPASTAAQKKKTPWWI ILI TLGLLQKGAGAT-VKITSSLVDYTFQQIIKD------SSTINVQAKFTSTAVDKPKTAPPDTVVIGFTAISPNLTKEGESSTVEWWI IFI VVTNLGTEDGDA--AFVAIRARMVAKI-MEKLASNVPLNVSTLAVANVTLLPF IGAPKDAIVKTHEIFYKAEPEPLQ-VPDVVPLWVVVI NLSALPKEESR-TINL YML----LNTEILKKDSS---SVIQFMARAKVKVEPALRVVEIANGNPEETLVVFEALHNLEPRGYVVGWIIAI OVVGRLDRGKSA --ILYVK-SLLWTE-TFMNKENQNHSYSLKSSASFNVIEF PY -KNLPIEDITNSTLVTTNVTWGIQPAPMPVPVWVI II DIYDLKEDEFV--LVEIFSR-LYTN-TLVDEKNP-G-GDISSIALARVTSTKYNLPHKPTLI---TAVSTNMNA IASEEGRDLPWWLYLL

\section{SVLGGLLLVAAVTVILYKVGFFKRKOIKDISAPDTTETTAL}

CILVAIL I A AVIVFIMYKKGFFKRKKMGEDEEEEELRKGDPEE

AACAGAL IFLLLVWLLYKCGFFNRNRPTDHSOERQPLRNGYHGDEHL

SLIVGILIFLLLAVILWKMGF FRRRYKEITEATEKNRKENEDGWDWVOKNO

AVILAGLLLLAYVLVFVMYTMGFFKRVRPPOEEO-EREQLQPHENGEGNSET

AIL IGLAILILLILLLWRCGFFKRNRP PTEHARERADRQPNAQYADSQSRYTSQDQYNQGRHGQML

Figure 4

$\alpha$ integrin alignments (carboxy terminal end of the molecules). See legend for Fig. 3. prior to the protostome/deuterostome split. Rather, it appears more likely that $\beta$ integrins have diverged independently in several bilaterian lineages.

\section{Expression of integrins during coral gastrulation}

RT-PCR analysis demonstrates that mRNAs encoding each of the coral integrins (AmItg $\alpha 1, A m I t g \beta 1$ and AmItg $\beta 2$ ) are present in eggs (Knack et al., unpublished data; [23]) and early developmental stages (Fig. 6) at relatively uni- form levels. No specific pattern could be detected via in situ hybridization prior to the prawn chip stage however, implying that the maternal mRNA is uniformly distributed until this time. In the early development of Acropora, a clear and specific in situ hybridization pattern for AmItg $\alpha 1$ is first seen in late prawn chip stage embryos (early gastrula) (Fig. 7A), the mRNA being localized in a characteristic pattern on the concave side of the flattened cell bilayer corresponding to Fig. 4b in Hayward et al. 

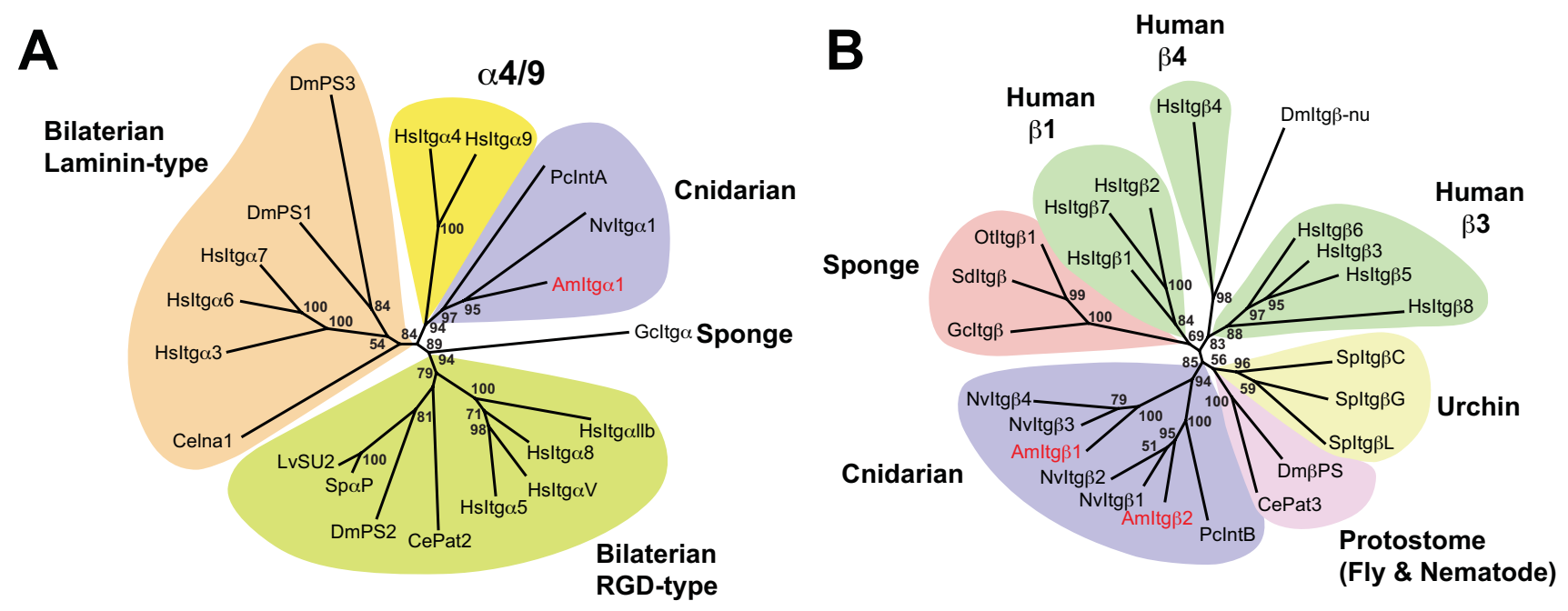

\section{Figure 5}

Maximum likelihood phylogenetic analysis of representative $\alpha$ and $\beta$ integrin proteins. Numbers at branch points indicate the percentage of 1000 bootstrap replicates supporting the topology shown (using MolPhy version 2.3; see [I4]). (A) $\alpha$ integrins. Whereas integrins from Bilateria group in a ligand specific manner, consistent with previous phylogenies, the cnidarian sequences form an independent clade, reflecting their early divergence. These groupings suggest that functional divergence of $\alpha$ integrins had already occurred in the Urbilateria. Sequences aligned, abbreviations, and accession numbers are: Lytechinus SU2 (LvSU2; AAC23572); Strongylocentrotus $\alpha$ P (SpaP; AAD55724); Drosophila aPS2 (DmPS2; PI2080); Human $\alpha 5$ (Hsltg $\alpha 5$;

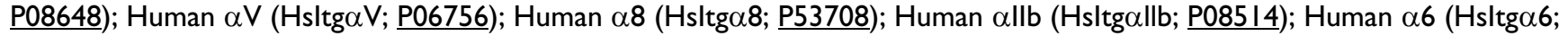

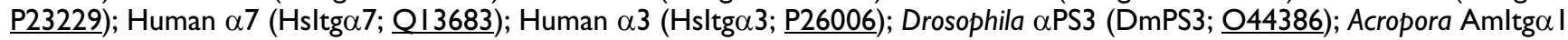

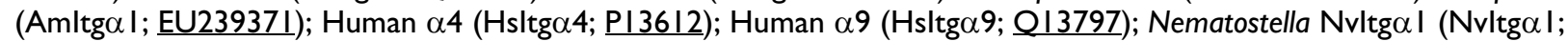
XP 00164I435); Caenorhabditis $\alpha$ Pat2 (CePat2; P34446); Drosophila aPSI (DmPSI; Q24247); Podocoryne IntA (PclntA; AAG25993); Caenorhabditis $\alpha$ lna I (Celna I; Q03600); Geodia $\alpha$ (Gcltg $\alpha$; CAA65943). (B) $\beta$ integrins. Major clades resolved here are consistent with previous phylogenies. The position of sequences within the cnidarian clade is consistent with orthology between Podocoryne IntB (PclntB) and Amltg $\beta 2$, and groups two Nematostella $\beta$ s with each Acropora $\beta$. Unlike the $\alpha$ integrins, the $\beta$ integrins appear to have diverged independently in several bilaterian lineages. Sequences aligned, abbreviations,

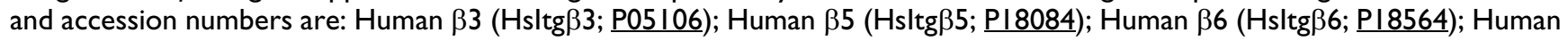
$\beta 2$ (Hsltg $\beta 2$; P05 I07); Human $\beta 7$ (Hsltg $\beta 7 ;$ P260I0); Human $\beta$ I (Hsltg $\beta$ I; P05556); Strongylocentrotus $\beta G$ (Urchin Spltg $\beta G ;$ AAB39739); Strongylocentrotus $\beta$ L (Spltg $\beta$ L; AAC28382); Strongylocentrotus $\beta C$ (Spltg $\beta C ;$ AAB39740); Drosophila $\beta P S$ (Dm $\beta P S$ PI I584); Caenorhabditis $\beta$ Pat3 (CePat3; Q27874); Acropora Amltg $\beta 2$ (Amltg $\beta 2$; EU239372); Nematostella $\beta$ I (Nvltg $\beta$ I; XP 00164I 468); Nematostella $\beta 2$ (Nvltg $\beta 2 ;$ XP 001627336); Podocoryne IntB (PclntB; AAG25994); Acropora Amltg $\beta$ I (Amltg $\beta$ I;

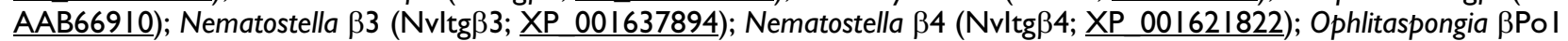
(Sponge Otltg $\beta$ I; AAB669II); Suberites $\beta$ (Sponge Sdltg $\beta$; CAB38I00); Geodia $\beta$ (Sponge Gcltg $\beta$; $\underline{\text { CAA7707I); Human } \beta 4}$

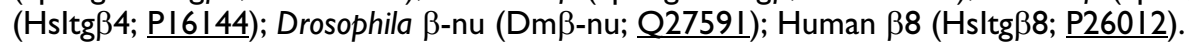

[14]. Expression is maintained in these presumptive endodermal cells as the concavity deepens and they are internalized. Staining remains strong in the presumptive endoderm until blastopore closure is complete (Fig. 7C), after which only weak endodermal staining of AmItg $\alpha 1$ is observed.

The early expression patterns of both AmItg $\beta 1$ and AmItg $\beta 2$ (Fig. 7) were broadly similar to that of AmItg $\alpha 1$, but with the following differences. First, it was only possible to visualize the localization of transcripts corresponding to the $\beta$ integrins at slightly later stages of development. Second, whereas AmItg $\alpha 1$ and AmItg $\beta 1$ transcripts were tightly restricted at the area of the blast- opore lip in early gastrulae (Fig 7B and 7E), AmItgß2 was also expressed more generally (Fig 7G-I).

\section{Discussion}

Whereas Reber-Muller et al. [11] hypothesized the presence of only single $\alpha$ and $\beta$ integrin subunits in cnidarians, the integrins identified to date in Acropora are likely to be only a subset of those present. Preliminary surveys of the genome of Nematostella imply that at least four $\beta$ integrins (two resembling AmItg $\beta 1$ and two more similar to AmItg $\beta 2$ ), and at least three distinct $\alpha$ types are present (data not shown). However, gene models for only one $\alpha$ integrin were sufficiently complete to allow its inclusion in the phylogenetic analyses shown as Fig 5A. The integrin 


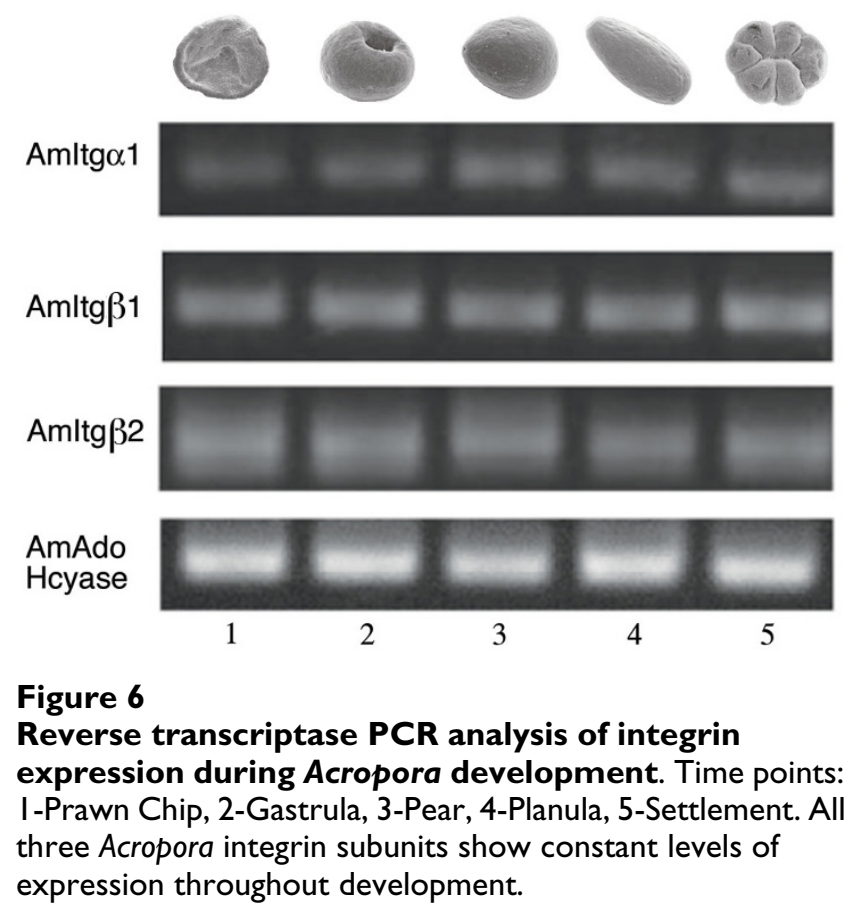

repertoire of this morphologically simple animal is therefore considerably more complex than that of Caenorhabditis (one $\beta$ and two $\alpha$ ), which has often been assumed to reflect the ancestral metazoan state.

Due to structural constraints on integrin proteins during evolution, phylogenetic analyses are likely to be complicated by homoplasy effects. The position of the cnidarian sequences in the $\alpha$ integrin phylogeny (Fig. 5A) is likely due to both the early divergence of the Cnidaria and homoplasy effects. Hence, in this case, phylogenetics is not informative as to ligand binding properties. It is likely that in cnidarians, as in higher animals, distinct $\alpha$ integrin types participate in binding to laminin and RGD-containing ligands, but functional analyses are required to verify this hypothesis.

Despite having a common pattern of cysteine loss, phylogenetic analyses (Fig 5B) indicate that AmItg $\beta 2$ is only distantly related to the vertebrate $\beta 4$-type. Those cysteine residues (positions 8 and 9) in the consensus absent from the Acropora and Podocoryne sequences form the $\mathrm{c} 8-\mathrm{c} 9$ loop of the $\beta$ A domain, which has been implicated in determining integrin-ligand specificity, specificity of $\alpha-\beta$ interactions, and signalling properties [24-28]. Whilst this loss has apparently occurred independently of that leading to the vertebrate $\beta 4$ type, it may result in common consequences for ligand and/or $\alpha$-subunit specificity.

The presence of maternal integrin mRNAs and their relatively uniform expression through development reported
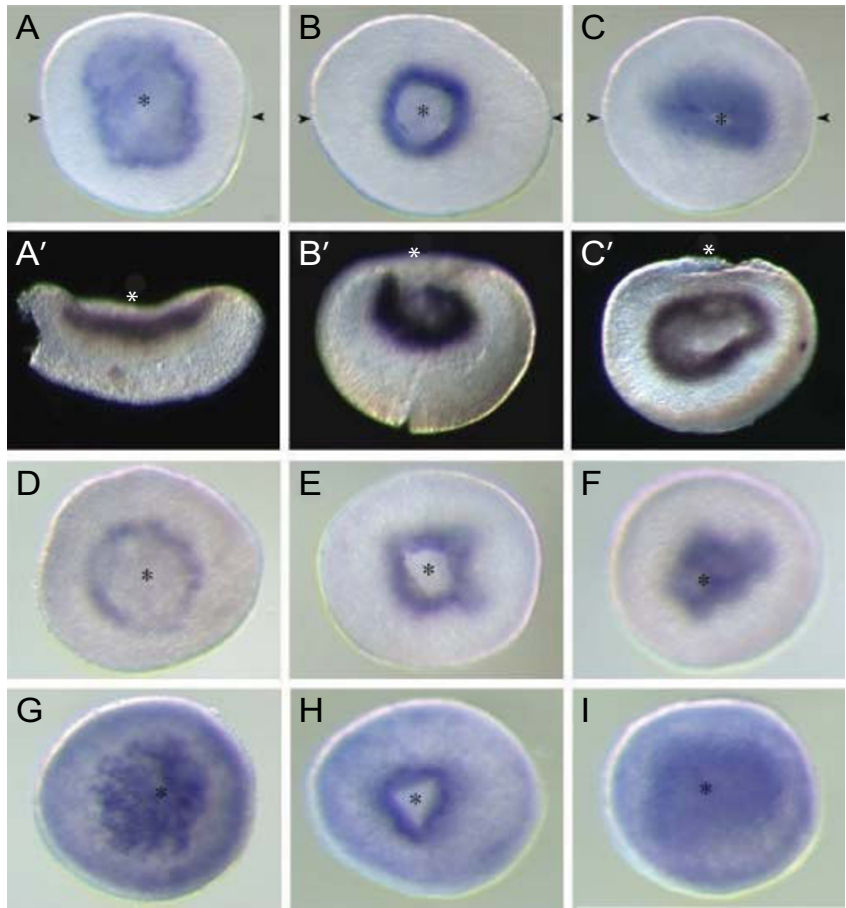

\section{Figure 7}

Comparison of Amltg $\alpha$ I, Amltg $\beta I$ and Amltg $\beta 2$ mRNA distribution patterns during gastrulation in Acropora. At the prawn chip stage, the Amltgol and Amltg $\beta \mid$ mRNAs are clearly restricted to one side of the flattened cell bilayer (A, $\left.A^{\prime}, D\right)$. During gastrulation, these mRNAs are tightly restricted to the area of the blastopore (asterisks) lip (B, B', C, C', E, F), and throughout development remain endodermal. The distribution of Amltg $\beta 2$ mRNA (G, $\mathrm{H}, \mathrm{I})$ is broadly similar to that of Amltgal, but is less tightly restricted, as indicated by weak general staining. Arrow heads in $A, B$, and $C$ indicate the plane of the sections shown as $A^{\prime}, B^{\prime}$ and $C^{\prime}$.

here for Acropora have precedents in Podocoryne [11] as well as in higher animals. Co-localization of mRNAs for $A m I t g \beta 1, A m I t g \beta 2$ and AmItg $\alpha 1$ suggests that either or both of the $\beta$ subunits associate with the $\alpha 1$ subunit. There are many precedents from bilaterians for RGD-type $\alpha$ associating with $\beta 1$-type $\beta$ s $(\alpha 5 \beta 1 ; \alpha \mathrm{V} \beta 1 ; \alpha 8 \beta 1$; PS2 $\beta$ PS). Of these, $\alpha 5 \beta 1$ and PS2 $\beta$ PS have been implicated as regulators of gastrulation in vertebrates $[29,30]$ and Drosophila [31] respectively. In Podocoryne, IntA is assumed to associate with IntB (a possible AmItg $\beta 2$ ortholog) since they are co-expressed in a wide variety of locations over a range of life cycle stages [11], suggesting that AmItg $\beta 2$ may associate with the AmItg $\alpha 1$ subunit.

Although integrins clearly play important roles in gastrulation in several animal groups, including vertebrates [29], Drosophila [31], and sea urchins [32], the interactions that have been demonstrated are heterogeneous 
with respect to both the ligands and types of integrins involved. The expression patterns of $\alpha$ and $\beta$ integrin genes observed in the coral are reminiscent of expression during both amphibian and sea urchin gastrulation. Modulation of integrin adhesion to the RGD domain of fibronectin plays a central role during Xenopus gastrulation [33], and in the sea urchin Lytechinus changes in laminin adhesion mediated by the epithelial $\alpha$ integrin $\alpha$ SU2 are likewise important [34]. In both the sea urchin and Xenopus, changes in integrin-mediated cell adhesion during gastrulation occur independently of transcription and translation. In Acropora, integrin mRNAs are present in eggs [23] and the extent to which those visualized in the presumptive endoderm reflect zygotic transcription is unknown.

The simplest interpretation of the patterns of integrin expression is that they reflect increases in cell adhesion in the presumptive endoderm. Expression on the concave side of the "fat prawn chip" stage embryo, which is essentially a flat bilayer of cells (for a description of gastrulation in Acropora, see [14]), suggests that integrin-based adhesion may constrain the presumptive endoderm whilst the cells of the presumptive ectoderm move upward and inward around them. Whilst the cells that will end up inside the embryo may adhere to each other more tightly than they do to the putative ectoderm, as is consistent with many forms of gastrulation, the inferred increased adhesion in the putative endoderm is not consistent with a typical epithelial to mesenchymal transition.

The early expression patterns of the $\alpha$ and $\beta$ integrins in Acropora are very similar to those of two transcription factors, snailA [15] and otxB [35]. Snail genes have central and conserved roles in gastrulation in Drosophila [36] and vertebrates [37] and members of the broader class of related genes are regulators of other epithelial to mesenchymal transitions (EMTs). Whilst the best understood means by which snail genes regulate cell adhesion is by acting as repressors of E-cadherin expression [36,38,39], in human epidermal keratinocytes the snail-related gene Slug (Snail2) is a repressor of $\alpha 3, \beta 1$ and $\beta 4$ integrin expression, leading to decreased cell-adhesion to fibronectin and laminin 5 [40]. Across the Bilateria, Otx genes are conserved anterior markers [41] and, whilst most Otx genes are expressed in the nervous system, evidence from a diverse range of metazoans suggests an ancient role as regulators of cell adhesion (eg. $[42,43])$. In Hydra, high levels of CnOtx expression correspond to regions where cells are undergoing rearrangements or movement [44]. Both snailA and otxB are thus candidate regulators of integrin expression in Acropora. Given the similarity of AmItg $\alpha 1$ to diverged RGD-type mammalian integrins, it will be of particular interest to examine the expression of ECM proteins containing fibronectin type
III domains during cnidarian gastrulation in parallel with adhesion studies. Candidates identified in Nematostella include predicted proteins similar to vertebrate usherin and titin, and a likely homolog of Drosophila sidekick.

\section{Conclusion}

Whilst one might expect morphologically simple metazoans to have a correspondingly basic integrin complement, comprising perhaps just two $\alpha$ s and a single $\beta$ subunit (as in Caenorhabditis elegans), the repertoire of these molecules in anthozoan cnidarians is considerably more complex. In the case of cnidarian $\alpha$ integrins, ligand specificity cannot be predicted by phylogenetic analysis, suggesting the possibility that specificity mechanisms arose independently in Cnidaria and Bilateria. During early development in Acropora, some of these adhesion/signalling molecules are expressed in patterns which parallel those of their amphibian and echinoderm counterparts, and which are inconsistent with gastrulation being a simple epithelial to mesenchymal transition.

\section{Methods}

\section{Sample collection and RNA extraction}

Developmentally staged Acropora millepora embryos were collected during annual spawning events. Embryos were staged based on Ball et al. (2002). Total RNA was extracted using RNAWIZ (Ambion) according to the manufacturer's protocol.

\section{RT-PCR analysis}

RNA was treated with DNase (Fermentas) to remove contaminating genomic DNA. Single stranded cDNA was synthesised using the First-strand cDNA Synthesis Kit (Amersham Biosciences, Piscataway, NJ) using $1 \mu \mathrm{g}$ of total RNA. One $\mu \mathrm{l}$ of this product was used as a polymerase chain reaction (PCR) template. For AmItg $\alpha 1$, primers AmItga1RTF (5'-GCCAATGAAACAGCTACG-3') and AmItga1RTR (5'-TTGTCTCCAGCCTTCAAC-3') were used to amplify a $130 \mathrm{bp}$ product. For $A m I t g \beta 2$, primers AmItgb2RTF (5'-TGGGCATTTGTGGTGTGAG-3') and AmItgb2RTR (5'-GCTTGTTCTGATGAGTGATGG-3') were used to amplify a 219 bp product. For AmItg $\beta 1$ (Brower et al. 1997), primers IB1RTF (5'-CTTGTGTTGCCACTTATGGCTT-3') and IB1RTR (5'-CTGCTACTTGCATTAACGCATC-3') were used to amplify a 144 bp product. The PCR protocol was $1 \mathrm{~min}$ at $94^{\circ} \mathrm{C}$, then 40 cycles of $0.5 \mathrm{~min}$ at $94^{\circ} \mathrm{C}$ (denaturation), $0.5 \mathrm{~min}$ at $50^{\circ} \mathrm{C}$ (annealing), $2 \mathrm{~min}$ at $72^{\circ} \mathrm{C}$ (extension), followed by an additional extension for $2 \mathrm{~min}$ at $72^{\circ} \mathrm{C}$.) As a control, primers ADH-F (5'AAGAAGACAAACATCAAGCCTCA-3') and ADH-R (5'CACATCCAAGGTTCACAAGACG-3') were used to amplify a portion of coral AdoHcyase (S-adenosyl-Lhomocysteine hydrolase) cDNA (unpublished data). 


\section{Whole mount in situ hybridization}

The basic procedures for fixation and hybridization with coral embryos were carried out as described [45]. Photographs were captured directly with a Spot digital camera. Digitised images were processed with Adobe Photoshop.

\section{Authors' contributions}

BAK was responsible for conducting DNA sequencing and RT-PCR analysis, and participated in both the in situ hybridisation experiments and preparing the manuscript. AI collected and prepared coral developmental stages, assisted in DNA sequencing and, with CS, carried out in situ hybridisation experiments and photographed the results. DCH was responsible for the initial identification of clones, assisted in sequence alignment and critically reviewed both the data and the manuscript. DJM conducted the phylogenetic analyses, and he and EEB drafted and reviewed the manuscript.

\section{Acknowledgements}

The authors thank the late Danny Brower for advice and critical input. This work was supported by grants from the Australian Research Council, both directly to DJM and EEB and via the Centre of Excellence for Coral Reef Studies and the Special Research Centre for the Molecular Genetics of Development. BAK acknowledges a James Cook University Postgraduate Research Scholarship, and Al and CS were supported by scholarships from the Okinawa International Exchange and Human Resources Development Council.

\section{References}

I. Hynes RO: Integrins: bidirectional, allosteric signaling machines. Cell 2002, I I 0:673-687.

2. De Arcangelis A, Georges-Labouesse E: Integrin and ECM functions: roles in vertebrate development. Trends Genet 2000, 16:389-395.

3. Marsden M, DeSimone DW: Integrin-ECM interactions regulate cadherin-dependent cell adhesion and are required for convergent extension in Xenopus. Curr Biol 2003, I3: I I82-I I9I.

4. consortium C: Genome sequence of the nematode $C$. elegans: a platform for investigating biology. Science 1998, 282:2012-2018.

5. Adams MD, Celniker SE, Holt RA, Evans CA, Gocayne JD, Amanatides PG, Scherer SE, Li PW, Hoskins RA, Galle RF, George RA, Lewis SE, Richards S, Ashburner M, Henderson SN, Sutton GG, Wortman JR, Yandell MD, Zhang Q, Chen LX, Brandon RC, Rogers YH, Blazej RG, Champe M, Pfeiffer BD, Wan KH, Doyle C, Baxter EG, Helt G, Nelson CR, Gabor GL, Abril JF, Agbayani A, An HJ, Andrews-Pfannkoch C, Baldwin D, Ballew RM, Basu A, Baxendale J, Bayraktaroglu L, Beasley EM, Beeson KY, Benos PV, Berman BP, Bhandari D, Bolshakov S, Borkova D, Botchan MR, Bouck J, Brokstein P, Brottier P, Burtis KC, Busam DA, Butler H, Cadieu E, Center A, Chandra I, Cherry JM, Cawley S, Dahlke C, Davenport LB, Davies P, de Pablos B, Delcher A, Deng Z, Mays AD, Dew I, Dietz SM, Dodson K, Doup LE, Downes M, Dugan-Rocha S, Dunkov BC, Dunn P, Durbin KJ, Evangelista CC, Ferraz C, Ferriera S, Fleischmann W, Fosler C, Gabrielian AE, Garg NS, Gelbart WM, Glasser K, Glodek A, Gong F, Gorrell JH, Gu Z, Guan $P$, Harris M, Harris NL, Harvey D, Heiman TJ, Hernandez JR, Houck J, Hostin D, Houston KA, Howland TJ, Wei MH, Ibegwam C, Jalali M, Kalush F, Karpen GH, Ke Z, Kennison JA, Ketchum KA, Kimmel BE, Kodira CD, Kraft C, Kravitz S, Kulp D, Lai Z, Lasko P, Lei Y, Levitsky AA, Li J, Li Z, Liang Y, Lin X, Liu X, Mattei B, McIntosh TC, McLeod MP, McPherson D, Merkulov G, Milshina NV, Mobarry C, Morris J, Moshrefi A, Mount SM, Moy M, Murphy B, Murphy L, Muzny DM, Nelson DL, Nelson DR, Nelson KA, Nixon K, Nusskern DR, Pacleb JM, Palazzolo M, Pittman GS, Pan S, Pollard J, Puri V, Reese MG, Reinert K, Remington K, Saunders RD, Scheeler F, Shen H, Shue BC, Siden-
Kiamos I, Simpson M, Skupski MP, Smith T, Spier E, Spradling AC, Stapleton M, Strong R, Sun E, Svirskas R, Tector C, Turner R, Venter E, Wang $A H$, Wang $X$, Wang ZY, Wassarman DA, Weinstock GM, Weissenbach J, Williams SM, WoodageT, Worley KC, Wu D, Yang S, Yao QA, Ye J, Yeh RF, Zaveri JS, Zhan M, Zhang G, Zhao Q, Zheng L, Zheng XH, Zhong FN, Zhong W, Zhou X, Zhu S, Zhu X, Smith HO, Gibbs RA, Myers EW, Rubin GM, Venter JC: The genome sequence of Drosophila melanogaster. Science 2000, 287:2185-2195.

6. Whittaker CA, Hynes RO: Distribution and evolution of von Willebrand/integrin A domains: widely dispersed domains with roles in cell adhesion and elsewhere. Mol Biol Cell 2002, 13:3369-3387.

7. Brower DL, Brower SM, Hayward DC, Ball EE: Molecular evolution of integrins: genes encoding integrin beta subunits from a coral and a sponge. Proc Natl Acad Sci U S A 1997, 94:9182-9187.

8. Pancer Z, Kruse M, Muller I, Muller WE: On the origin of Metazoan adhesion receptors: cloning of integrin alpha subunit from the sponge Geodia cydonium. Mol Biol Evol 1997, 14:391-398.

9. Wimmer W, Perovic S, Kruse M, Schroder HC, Krasko A, Batel R, Muller WE: Origin of the integrin-mediated signal transduction. Functional studies with cell cultures from the sponge Suberites domuncula. EurJ Biochem 1999, 260:156-165.

10. Muller WE: Origin of metazoan adhesion molecules and adhesion receptors as deduced from cDNA analyses in the marine sponge Geodia cydonium: a review. Cell Tissue Res 1997, 289:383-395.

II. Reber-Muller S, Studer R, Muller P, Yanze N, Schmid V: Integrin and talin in the jellyfish Podocoryne carnea. Cell Biol Int 200I, 25:753-769.

12. Ball EE, Hayward DC, Saint R, Miller DJ: A simple plan--cnidarians and the origins of developmental mechanisms. Nat Rev Genet 2004, 5:567-577.

13. Technau U, Rudd S, Maxwell P, Gordon PM, Saina M, Grasso LC, Hayward DC, Sensen CW, Saint R, Holstein TW, Ball EE, Miller DJ: Maintenance of ancestral complexity and non-metazoan genes in two basal cnidarians. Trends Genet 2005, 21:633-639.

14. Hayward DC, Samuel G, Pontynen PC, Catmull J, Saint R, Miller DJ, Ball EE: Localized expression of a dpp/BMP2/4 ortholog in a coral embryo. Proc Natl Acad Sci U S A 2002, 99:8106-8III.

15. Hayward DC, Miller DJ, Ball EE: snail expression during embryonic development of the coral Acropora: blurring the diploblast/triploblast divide? Dev Genes Evol 2004, 2 14:257-260.

16. Kortschak RD, Samuel G, Saint R, Miller DJ: EST analysis of the cnidarian Acropora millepora reveals extensive gene loss and rapid sequence divergence in the model invertebrates. Curr Biol 2003, 13:2190-2195.

17. Tozer EC, Hughes PE, Loftus JC: Ligand binding and affinity modulation of integrins. Biochem Cell Biol 1996, 74:785-798.

18. Tuckwell DS, Brass A, Humphries MJ: Homology modelling of integrin EF-hands. Evidence for widespread use of a conserved cation-binding site. Biochem J 1992, 285 ( Pt I ):325-33 I.

19. Adachi J, Hasegawa M: Instability of quartet analyses of molecular sequence data by the maximum likelihood method: the Cetacea/Artiodactyla relationships. Mol Phylogenet Evol 1996, 6:72-76

20. Hughes $A L$ : Evolution of the integrin alpha and beta protein families. J Mol Evol 200I, 52:63-72.

21. Ewan R, Huxley-Jones J, Mould AP, Humphries MJ, Robertson DL, Boot-Handford RP: The integrins of the urochordate Ciona intestinalis provide novel insights into the molecular evolution of the vertebrate integrin family. BMC Evol Biol 2005, 5:31.

22. Huhtala M, Heino J, Casciari D, de Luise A, Johnson MS: Integrin evolution: insights from ascidian and teleost fish genomes. Matrix Biol 2005, 24:83-95.

23. Iguchi A, Marquez LM, Knack B, Shinzato C, van Oppen MJ, Willis BL, Hardie K, Catmull J, Miller DJ: Apparent Involvement of a beta I Type Integrin in Coral Fertilization. Mar Biotechnol (NY) 2007.

24. Bunch TA, Miller SW, Brower DL: Analysis of the Drosophila betaPS subunit indicates that regulation of integrin activity is a primal function of the C8-C9 loop. Exp Cell Res 2004, 294: I 18-129.

25. Takagi J, Kamata T, Meredith J, Puzon-McLaughlin W, Takada Y: Changing ligand specificities of alphavbeta $I$ and alphavbeta 3 
integrins by swapping a short diverse sequence of the beta subunit. J Biol Chem 1997, 272:19794-19800.

26. Lin CS, Chen $Y$, Huynh T, Kramer R: Identification of the human alpha6 integrin gene promoter. DNA Cell Biol 1997, 16:929-937.

27. Takagi J, DeBottis DP, Erickson HP, Springer TA: The role of the specificity-determining loop of the integrin beta subunit Ilike domain in autonomous expression, association with the alpha subunit, and ligand binding. Biochemistry 2002, $41: 4339-4347$.

28. Miao H, Li S, Hu YL, Yuan S, Zhao Y, Chen BP, Puzon-McLaughlin W, Tarui T, Shyy JY, Takada Y, Usami S, Chien S: Differential regulation of Rho GTPases by betal and beta 3 integrins: the role of an extracellular domain of integrin in intracellular signaling. J Cell Sci 2002, I I 5:21 99-2206.

29. Davidson LA, Hoffstrom BG, Keller R, DeSimone DW: Mesendoderm extension and mantle closure in Xenopus laevis gastrulation: combined roles for integrin alpha(5)beta(I), fibronectin, and tissue geometry. Dev Biol 2002, 242:109-129.

30. Whittaker CA, DeSimone DW: Integrin alpha subunit mRNAs are differentially expressed in early Xenopus embryos. Development 1993, II 7: 1239-1249.

31. Roote CE, Zusman S: Functions for PS integrins in tissue adhesion, migration, and shape changes during early embryonic development in Drosophila. Dev Biol 1995, 169:322-336.

32. Marsden M, Burke RD: The betaL integrin subunit is necessary for gastrulation in sea urchin embryos. Dev Biol 1998, 203: 134-I 48.

33. Ramos JW, DeSimone DW: Xenopus embryonic cell adhesion to fibronectin: position-specific activation of RGD/synergy site-dependent migratory behavior at gastrulation. J Cell Biol 1996, I34:227-240.

34. Hertzler PL, McClay DR: alphaSU2, an epithelial integrin that binds laminin in the sea urchin embryo. Dev Biol 1999, 207: $1-13$.

35. de Jong DM, Hislop NR, Hayward DC, Reece-Hoyes JS, Pontynen PC, Ball EE, Miller D]: Components of both major axial patterning systems of the Bilateria are differentially expressed along the primary axis of a 'radiate' animal, the anthozoan cnidarian Acropora millepora. Dev Biol 2006, 298:632-643.

36. Ip YT, Gridley T: Cell movements during gastrulation: snail dependent and independent pathways. Curr Opin Genet Dev 2002, I 2:423-429.

37. Carver EA, Jiang R, Lan Y, Oram KF, Gridley $T$ : The mouse snail gene encodes a key regulator of the epithelial-mesenchymal transition. Mol Cell Biol 200I, 21:8I84-8I88.

38. Barrallo-Gimeno A, Nieto MA: The Snail genes as inducers of cell movement and survival: implications in development and cancer. Development 2005, |32:3|5|-3|6|.

39. Nieto MA: The snail superfamily of zinc-finger transcription factors. Nat Rev Mol Cell Biol 2002, 3:155-166.

40. Turner FE, Broad S, Khanim FL, Jeanes A, Talma S, Hughes S, Tselepis $C$, Hotchin NA: Slug regulates integrin expression and cell proliferation in human epidermal keratinocytes. I Biol Chem 2006, 28I:2|32|-2|33|.

4I. Lichtneckert R, Reichert $H$ : Insights into the urbilaterian brain: conserved genetic patterning mechanisms in insect and vertebrate brain development. Heredity 2005, 94:465-477.

42. Bellipanni G, Murakami T, Doerre OG, Andermann P, Weinberg ES: Expression of Otx homeodomain proteins induces cell aggregation in developing zebrafish embryos. Dev Biol 2000, 223:339-353.

43. Perea-Gomez A, Lawson KA, Rhinn M, Zakin L, Brulet P, Mazan S, Ang SL: Otx2 is required for visceral endoderm movement and for the restriction of posterior signals in the epiblast of the mouse embryo. Development 200I, 128:753-765.

44. Smith KM, Gee L, Blitz IL, Bode HR: CnOtx, a member of the Otx gene family, has a role in cell movement in hydra. Dev Biol 1999, 21 2:392-404.

45. Hayward DC, Catmull J, Reece-Hoyes JS, Berghammer H, Dodd H, Hann SJ, Miller DJ, Ball EE: Gene structure and larval expression of cnox-2Am from the coral Acropora millepora. Dev Genes Evol 200I, $211: 10-19$ Publish with Biomed Central and every
scientist can read your work free of charge

"BioMed Central will be the most significant development for disseminating the results of biomedical research in our lifetime. "

Sir Paul Nurse, Cancer Research UK

Your research papers will be:

- available free of charge to the entire biomedical community

- peer reviewed and published immediately upon acceptance

- cited in PubMed and archived on PubMed Central

- yours - you keep the copyright

Submit your manuscript here:

http://www.biomedcentral.com/info/publishing_adv.asp
BioMedcentral 